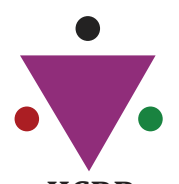

IJCRR

Section: Healthcare Sci. Journal Impact Factor: $6.1(2018)$ ICV: 90.90 (2018)

\title{
Comparative Efficacy of 3Dimensional (3D) Cell Culture Organoids Vs 2Dimensional (2D) Cell Cultures Vs Experimental Animal Models In Disease modeling, Drug development, And Drug Toxicity Testing
}

\author{
Santenna Chenchula ${ }^{1}$, Sunil Kumar ${ }^{1}$, Shoban Babu V' \\ 'Department of Pharmacology, Demonstrator, AllMS, Bhopal; '2Department of Pharmacology, Assistant Professor, AllMS, Jodhpur.
}

\section{ABSTRACT}

Historically animal studies and 2D cell culture models have been strengthening biomedical and pharmaceutical research, with many limitations. Currently, new drug development for many diseases like cancer is an important necessity. An organoid is a miniaturized version of an organ produced in vitro that shows realistic micro-anatomy, is capable of self-renewal and self-organization and exhibits similar functionality as the tissue of origin. While their size is small (typically $<3 \mathrm{~mm}$ in diameter), organoids are stable model systems of organs and tissues that are amenable to long-term cultivation and manipulation. They are classified into those that are tissue-derived and those that are stem cell-derived. They help in both in vivo and in vitro investigation and represent one of the latest innovations in the research for a model to recapitulate the physiologic processes of whole organisms. They reduce experimental complexity, and are compliant to real-time imaging techniques, and more importantly, they enable the study of aspects of human development and disease, drug toxicity in a clear fashion that is not easily or correctly modelled in animals and 2D cell cultures. However 3D organoids have also had some limitations like vascularity, inflammatory system, etc. Despite these limitations, it is evident that organoids have great potential to revolutionize the way we approach disease modelling, drug discovery, and toxicology.

Key Words: 3D organoids, 2D cell culture, Drug discovery, Organ toxicity, High-throughput screening

\section{INTRODUCTION}

Traditionally animal studies using rats and mice etc., and 2D cell culture models have been used over the past decades in the field of biomedical research. Both these models are extremely helpful in disease modelling prior to the advent of the three-dimensional (3D) cell culture organoid technology. Nude and severe combined immunodeficiency (SCID) mice are immunodeficient mice, which are most commonly used as conferee of human cells or tissues as they accept foreign tissues or cells relatively easily due to a lack of host immunity. Another variety of rodents are humanized mice; this variety of mice lacks an innate immune system because these varieties completely procreated with human immune cells by hematopoietic stem cell transplantation [1]. Such humanized mouse models are particularly useful to model disease pathology and to allow for the assessment of potential therapeutic candidates. They provide a systemic environment to study disease pathology due to the presence of an intact immune system and blood circulation, which are essentially impossible in 2D models. Many studies have been succeeded in providing new insight into disease pathogenesis using humanized rodents in modelling human diseases. However, there are many raising questions on the pertinence of using mouse models to study human diseases [2]. 2D cell cultures used to study different cell types, along with drug screening

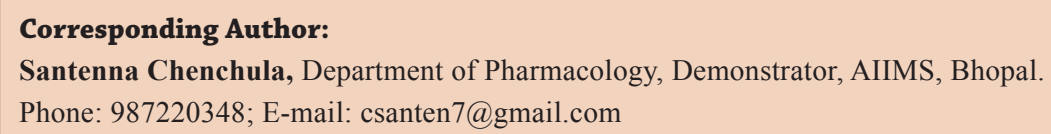


and testing. Usually, it contains the monolayer system which allows cell growth over a polyester or glass flat surface presenting a medium that feeds the growing cell population [3].Endless biological breakthroughs occurred through 2D cell culture research(quote few examples). However these 2D cell culture models have limitations due to their simplicity, lack of tissue-specific architecture, mechanical and biochemical cues, and cell-to-cell and cell-to-matrix interactions, so this model can't accurately depict and simulate the rich environment and complex processes observed in vivo such as cell signalling, chemistry or geometry, which makes them relatively poor models to predict drug responses for certain diseases like cancer [4]. As a result, data gathered with 2D cell culture methods could be non-predictive or misleading. Use of murine animal models for disease modelling, drug testing, and therapeutic development is not only costly and time-consuming but may not mimic biological responses in humans due to species differences. Considering all these limitations of animal studies and 2D cell culture system are not effective in disease modelling and drug research. However 3D cell culture organoids serve to overcome the challenges faced by 2D culture and animal disease models. Nonetheless, such modelling of human diseases in 3D cell culture organoids may still need to be validated in vivo, ultimately using a humanized mouse model.

\section{Dimensional (3D) cell culture Organoids}

An organoid is "a collection of organ-specific cell types that develops from stem cells or organ progenitors and selforganizes through cell sorting and spatially restricted lineage commitment in a manner similar to in vivo.An organoid is a miniaturized version of an organ produced in vitro that shows realistic micro-anatomy, is capable of self-renewal and self-organization and exhibits similar functionality as the tissue of origin. While their size is small (typically $\leq 3$ $\mathrm{mm}$ in diameter), organoids are stable model systems of organs and tissues that are amenable to long-term cultivation and manipulation. 3D cell culture organoid constructs composed of multiple cell types that originate from stem cells by means of self-organization and is capable of simulating the architecture and functionality of native organs $[5,6]$.They constitute a rapidly expanding family of dish-based, 3D developing tissues that show sensible microanatomy. They are generated with the use of somatic cells, adult stem cells (progenitor cells), or pluripotent stem cells. They are similar to their original organs, so they hold many advantages over traditional two-dimensional cultures and even animal models for use in medical research and the development of new treatments.

3D cell culture Organoids are of two types, tissue and stem cell organoids, depending on how the organ buds are formed. Stem cell-derived organoids may only recapitulate the first few months of development, but not the stages beyond.
Therefore they potentially lack some cell types of interest for biomedical research. However, tissue organoids generated from the isolated adult stem or progenitor cells are resected fragments of organ tissues are more suitable in the field of biomedical and pharmaceutical research. Till now there are many in vitro organoids have been established to resemble various tissues, including functional organoids for thyroid, pancreas, liver, stomach, intestine, cerebral cortex, pituitary, etc.[7,8].These 3D cell culture organoid tissues help in both in vivo and in vitro investigation and represent one of the latest innovations in the research for a model to recapitulate the physiologic processes of whole organisms $[9,10,11]$. They display near-physiologic cellular composition and behaviours. Compared to animal models, 3D organoids will reduce experimental complexity, and are compliant to realtime imaging techniques, and, more importantly, they enable the study of aspects of human development and disease that are not easily or correctly modelled in animals (Fig 1, Table 1).In contrast to conventional $2 \mathrm{D}$ methods, cells cultured in a 3D cell culture organoids may exhibit unique biochemical and morphological features, which are similar to their corresponding tissues in vivo. Nowadays organoids use in biomedical research progressing for the modelling of a disease, screening of new drugs, and pathophysiological characterization.Although organoid technology is expanded in the biomedical field, yet it is still in its infancy. There are many practical challenges to overcome before it can be widely implemented in disease modeling, drug discovery, and toxicological applications (Table 1). Still, there are many advantages of $3 \mathrm{~d}$ organoids over animal experimental models and 2D cell culture organoids (Figure 1) (Table 1).

\section{Disease modelling}

Disease modelling plays an important role in drug discovery. Animal model studies of both in vitro and in vivo were commonly employed for disease modelling. 3D cell cultures Organoids Bridge the gap between 2D cell culture and in vivo animal model studies. A range of 3D cell cultures has been applied until now to understand the mechanisms of different diseases.3D cell cultures organoids may provide more fundamental insights into development, homeostasis, and pathogenesis and may offer new translational approaches for the diagnosis and treatment of disease. For example to study the human brain, develop cerebral organoids to recapitulate human-specific neurogenic processes [12]. Human cerebral organoids have been grown in a microfabricated compartment that allows long-term in situ imaging. This system has been used to model the physics of cortical folding and to study the mechanism underlying lissencephaly, which is caused by mutations in LIS1 [13].

Cerebral organoids have also been used to show that the Zika virus preferentially infects neural progenitors and reduces their multiplication and viability, which may, in turn, 
be a cause of Zika virus-associated microcephaly [14]. This in vitro reflection of in vivo phenotype helps to investigate disease mechanisms and develop new treatment strategies in less time, and also reduces experimental animal use.

Recently 3D cell culture technologies generated novel 3D neural cell culture models that recapitulate Alzheimer's disease (AD) pathology including robust $\mathrm{A} \beta$ deposition and A $\beta$-driven NFT-like tau pathology [15]. These novel organoids of $\mathrm{AD}$ hold a promise for a novel platform that can be used for mechanism studies in human brain-like environment and highly selective drug screening. In the past decade, $\mathrm{AD}$ transgenic mice have been used as a standard preclinical model for testing candidate AD drug targets. The test compounds are tested in $\mathrm{AD}$ transgenic mice with multiple doses to explore their potential toxicity and the impact on $\mathrm{AD}$ pathology, including pathogenic $A \beta$ accumulation, $p$-tau accumulation and the behavioural and memory deficits. This process takes more than 2-3 years and is relatively expensive. The only small number of test compounds can pass through this process, and a majority of Alzheimer drug targets which showed efficacy in all the biochemical, cell culture and Alzheimer disease transgenic models, have failed to show efficacy in human clinical trials [14]. Even 3D culture models of Alzheimer disease are relatively cheaper and faster (6-10 weeks for our 3D culture model; 12 weeks for 3D organoid models) as compared to AD transgenic mouse model [16]. A $3 \mathrm{D}$ cell culture organoid technology has integrated with other technologies, including genome editing, single-cell genomics, live imaging, and microfluidics, thus providing new insights into developmental processes and disease pathogenesis as well as enabling translational approaches to the diagnosis and treatment of disease.

Neural cortical organoids which are induced from human induced pluripotent stem cells (hiPSCs) obtained from individuals with severe idiopathic autism spectrum disorder (ASD) are used to model autism. These cortical organoids exhibit a decreased cell cycle duration, indicative of flustered cell cycle potential, and showed an overproduction of GABAergic inhibitory neurons, providing critical insight into the pathogenesis of ASD. The success of cortical organoids in modelling autism is largely due to the ability to model embryonic telencephalic development seen in the third trimester of human development, as well as recapturing the regulatory networks of GABAergic neuron production. The authors identified gene expression of FOXG1 could potentially be used as a biomarker of severe autism.Irregularity of FOXG1 gene predominant in these cortical organoids provides an understanding of the alterations in the dynamics of brain growth and differentiated neurons [17].

\section{Cancer modeling}

Treating cancer has been challenging since long back, due to the complexity and heterogeneity of tumours, leading to resistance to chemotherapy. This complexity is partly due to the interaction between the tumor and its microenvironment [18]. The tumor microenvironment (TME) mainly consists of different non-cancer cell types and their stroma, such as fibroblasts, immune cells like lymphocytes and macrophages, mesenchymal cells, and endothelial cells (EC), which all have a specific role in the physiology, structure, and function of the tumour. In cancer research, well-established and characterized 2D cancer cell culture has massively contributed to the understanding of carcinogenesis from cell proliferation and migration to drug discovery [18].Yet cancer remains a complex disease that is still not fully understood, especially due to its close interactions with its surrounding cells.2D cultures generally fail to translate accurately the natural in vivo setting. When cells are cultured in $2 \mathrm{D}$, they grow as monolayers, which lead to polarized cell adhesion and two-dimensional contact with neighbouring cells. This physical characteristic allows them to receive a homogeneous amount of nutrients and growth factors from the media, resulting in abnormal cell spreading, an unrealistic distribution of cell surface receptors, and selection for specific cell sub-populations best adapted to $2 \mathrm{D}$ in vitro growth. There are some shortcomings in animal models like; slow growth rates of many engrafted primary cancers in patient-derived xenografts (PDX) models, and tumour heterogeneity [19]. So all these limitations of 2D cell culture and animal studies have encouraged the development of many 3D cell culture methods to better translate the complex pathophysiological features of the TME in vitro.Emerged3D cell culture organoid models have gained much popularity in studying tumor biology because standard 2D models are ineffective to answer questions regarding indolent disease, metastatic colonization, dormancy, relapse, and the rapid evolution of drug resistance. Different types of the Primary tumor organoids of colon, prostate, breast, ovarian and pancreas are successfully developed [20]. These are called "tumouroids", and they emerged as preclinical models that have the potential to predict an individual patient's response to treatment. Library of an organoid representing different grades of colorectal tumors revealed a decreased dependence on niche factors along with the transition from normal tissue to adenoma to carcinoma [20]. Niche factor dependency is found to be primarily associated with the genetic makeup of a tumour. So these tumoroids are a means of linking cancer-related genomic data to tumour biology and can provide a substrate for drug screening and personalized treatment.

\section{Drugis discovery}

Drug discovery is heavily reliant on high-throughput screening (HTS); the process of identifying hits by testing a large number of diverse chemical structures against disease targets and is characterized by its simplicity, efficacy, low cost per assay, and high efficiency [21]. 3D cell cultures organoids have an important role to discover novel mechanisms and 
targets to accelerate lead identification and validation, as the gene expression patterns found in 3D models are closer to in vivo, compared to 2D monolayer models [22]. Drugs identified on the basis of 2D-cultured cell lines give aberrant responses, and they have a considerable impact on the drug selection compared to drugs identified on the 3D cell culture organoid tissues. For instance, cancer cells grown as a monolayer have a deregulated cell cycle, often doubling every $24 \mathrm{~h}$, while tumours in vivo typically show only a few percents of actively cycling cells and only have a marginally higher rate of proliferation compared with healthy tissue. As a result, cancer drugs selected on the basis of arresting proliferation in culture often do little in vivo, or, if they do, will also show adverse effects in healthy tissue[23].

\section{Drug toxicity testing}

Every year billions are spent on developing targets identified from in vitro systems through to Phase III trials in patients. The vast majority of these compounds fail due to either unacceptable toxicities or limited efficacy in humans. So traditional 2D cell systems are ineffective in predicting clinical responses. Organ toxicity is one of the common limitations of drug development failures and withdrawals after marketing [24]. Among all organ toxicities currently, liver, heart, kidney, and brain toxicity account for more than $70 \%$ of drug attrition and withdrawal from the market. Renal and hepatotoxicity are the very common leading cause of drug attrition, is often accompanied by dysfunction in the bile transport system. Current toxicology screening tests that use cell lines and animal models often do not predict complete adverse effects profile of a new drug in human beings, essentially in whom renal and hepatic toxicities are among the most common.3D cell culture Organoids can also be useful in drug screening applications for assessing efficacy and safety, they are powerful in assessing drug-induced toxicity. They may offer more accurate means of toxicity prediction than animal models. Organ buds of brain, liver, heart, and kidney can be used to assess drug toxicity [25]. For instance, a brain organoid which was produced by combining human embryonic stem cell (ESC) derived neural progenitor cells, endothelial cells, Mesenchymal stem cells(MSCs), and microglia/macrophage precursors on chemically defined polyethylene glycol hydrogels. Machine learning was used to build a predictive model from changes in global gene expression when being exposed to 60 training compounds ( 34 toxic and 26 nontoxic chemicals) [26]. This model was then used to correctly classify 9 additional chemicals in a blinded trial.

The hepatic bi-progenitor cell line HepaRG is a unique cell line showing great plasticity, which differentiates to both canaliculae-like and hepatocyte-like cells. Human liver organoids obtained using HepaRG cell line; a terminally differentiated hepatic cell line derived from a human hepatic progenitor cell line, and have been shown to produce human- specific metabolites [27]. This is very useful because generally, the human liver metabolizes drugs in a manner distinct from animal liver. Of note, these HepaRG 3D organoid cultures are more sensitive to paracetamol or rosiglitazone-induced toxicity but less sensitive to troglitazone-induced toxicity than the 2D cultures. Kidney organ buds from human iPSC cells were found to differentially apoptosis in response to cisplatin, a nephrotoxicant, showing such organoids represent powerful models of the human organ for drug-induced nephrotoxicity. Favourably organoids of the kidney have been shown to recapitulate the nephrotoxic effects of cisplatin and gentamicin [28, 29, 30]. Also, organoids have advantages include their genetic stability and scalability for high-throughput screens e.g. Like human nephron progenitor cells have a nearly unlimited ability to self-renew in threedimensional culture, which could be beneficial for standardization of nephrotoxicity screens [5].

Organs-on-chips and other 3D cell culture models were the food and drug administration (FDA) approved to evaluate drug-induced toxicity [31]. Recently the FDA has started testing three-dimensional "liver-on-a-chip" constructs to screen for the hepatic toxicity of compounds used in food additives, nutritional supplements, and cosmetics. Hearton-a-chip devices were useful for assessing drug-induced cardiotoxicity. The lung-on-chip model consists of channels lined by closely apposed layers of human pulmonary epithelial and endothelial cells that experience air and fluid flow, enabling the detection of drug toxicity-induced pulmonary oedema observed in human cancer patients treated with interleukin-2 at similar doses and over the same time frame. They were also found that both angiopoietin-1 and GSK2193874 (a transient receptor potential vanilloid 4 ion channel inhibitor) were effective at preventing the drug toxicity-induced pulmonary oedema [32].

\section{Cell-based therapy using Intestinal organoids}

Cystic fibrosis is an autosomal recessive disease caused by the cystic fibrosis transmembrane-conductor regulator (CFTR) gene mutation. Colon organoids are generated from patients with cystic fibrosis. Genome editing using CRISPR/ Cas9 (clustered regularly interspaced short palindromic repeat/associated protein 9) has been used to correct mutations in CFTR and to restore the functionality of the CFTR protein in colon organoids derived from patients with cystic fibrosis [33]. However such studies suggest that organoids may be a source of cells in future approaches to cell therapy.

\section{Limitations of 3D cell culture organoids}

Although organoids have a wide range of potential applications, the current version still represents a somewhat rough model, and researchers still grapple with obstacles of this technology. Firstly the present 3D organoid systems have reproducibility limitations as there is little or no control over 
how cells self-organize into the organoids [34]. So, these protocols are unable to produce exact replications of organoid spheres of the same dimensions (size and shape), cellular composition, phenotypic and molecular characteristics. The "Tissues In a Dish" contains only an epithelial layer without native microenvironment including surrounding mesenchyme, immune cells, nervous system, or muscular layer .to overcome this limitation, the stem cell niche was proposed as a major aspect in which scientists can manipulate to improve the reproducibility of the organoids obtained

Vascularization is another important limitation of 3D organoids which is important for the supply of oxygen and nutrients to be supplied throughout the mass, encouraging better development of cells into tissue-like structures and for the cells that are within the mass to be able to survive and function as well as cells on the periphery. Even a vascularized organoid only will be able to capture the drug uptake, circulation, and metabolism that occur in the body [35].

3D organoid models are unable to produce the process of inflammation that occurs in vivo, which involves a multitude of cell types (endothelial cells, monocytes, macrophages, leukocytes) and cellular processes (leukocyte-endothelial cell adhesion, leukocyte extravasation and transmigration, and monocyte to macrophage differentiation) [36]. Blood is very crucial for the body's inflammatory response by serving as the carrier medium for immune cells. These immune cells eventually move to sites of injury through chemo-attraction and interact with blood vessel walls (through adhesion molecules presented by endothelial cells) before extravasating to inflamed loci. Therefore, in order to mimic inflammation in $3 \mathrm{D}$ in vitro models, vascularization has to first be established, blood perfusion has to occur, and immune cell types must be present. A 3D model that can successfully incorporate such an inflammation niche will be an extremely powerful tool for studying atherosclerotic vascular diseases, inflammatory skin diseases, interstitial nephritis, and even inflammatory bowel disease. In fact, humanized mouse models provide a systemic environment to holistically study disease pathology due to the presence of an intact immune system and blood circulation, which are essentially impossible in 2D and 3D models.

\section{DISCUSSION AND CONCLUSION}

3D cell culture organoids have been a major advancement to increase productivity and newer drugs development in pharmaceutical research and development. They hold great potential as a tool in new drug discovery - ranging from disease modelling to drug discovery, drug toxicity testing and as a new type of therapeutics/replacement therapy that may transform our lives. But these 3D cell culture organoids also have some limitations like lack of reproducibility, vascular- ity and lack of inflammation niche .So in future 3D cell models have to overcome these challenges and more research developments are needed in 3D organoid cell models, and will no doubt bring them closer to reaching these limitations in the biomedical and pharmaceutical field of research.

\section{ACKNOWLEDGEMENT}

Authors acknowledge the immense help received from the scholars whose articles are cited and included in references to this manuscript. The authors are also grateful to authors / editors / publishers of all those articles, journals and books from where the literature for this article has been reviewed and discussed.

\section{REFERENCES}

1. Shultz, L.D.; Brehm, M.A.; Garcia-Martinez, J.V.; Greiner, D.L. Humanized mice for immune system investigation: Progress, promise, and challenges. Nat. Rev. Immunol. 2012;12:786-98.

2. Mestas, J.; Hughes, C.C. Of mice and not men: Differences between mouse and human immunology. J. Immunol.2004; 172:2731-8.

3. Haycock J.W. 3D Cell Culture: A Review of Current Approaches and Techniques. 3D Cell Culture. Methods in Molecular Biology (Methods and Protocols).2011;695:1-15

4. Ravi M1, Paramesh V, Kaviya SR, Anuradha E, Solomon FD.3D cell culture systems: advantages and applications. J Cell Physiol. 2015 Jan;230:16-26

5. Duarte AA, Gogola E, Sachs N, et al. BRCA-deficient mouse mammary tumor organoids to study cancer-drug resistance. Nat Methods 2018; 15: 134-40.

6. Li Z, Araoka T, Wu J, et al. 3D culture supports long-term expansion of mouse and human nephrogenic progenitors. CellStem Cell 2016:516-29.

7. Mo Li, Juan C.Izpisua Belmonte. Organoids Preclinical Models of Human Disease. N Engl J Med 2019; 380:569-79.

8. Ye Fang1 and Richard M. Eglen. Three-Dimensional Cell Cultures in Drug Discovery and Development. SLAS DISCOVERY: Advancing Life Sciences R\&D: 2017; 456-72

9. Sato T, Vries RG, Snippert HJ, et al.Single Lgr5 stem cells build crypt-villus structures in vitro without a mesenchymal niche. Nature 2009;459: 262-5.

10. Barker N, van Es JH, Kuipers J, et al. Identification of stem cells in small intestine and colon by marker gene Lgr5. Nature 2007;449: 1003-7.

11. Hofmann C, Obermeier F, Artinger M, et al. Cell-cell contacts prevent anoikis in primary human colonic epithelial cells. Gastroenterology 2007;132: 587-600.

12. Lancaster MA, Renner M, Martin CA, et al. Cerebral organoids model human brain development and microcephaly. Nature2013; 501: 373-9.

13. Karzbrun E, Kshirsagar A, Cohen SR, Hanna JH, Reiner O. Human brain organoids a chip reveal the physics of folding. Nat Phys 2018; 14:515-22.

14. Dang J, Tiwari SK, Lichinchi G, et al.Zika virus depletes neural progenitors inhuman cerebral organoids through activation of the innate immune receptorTLR3. Cell Stem Cell 2016; 19: 258-65. 
15. Se Hoon Choi, Young Hye Kim, Luisa Quinti, Rudolph E. Tanzi, Doo Yeon Kim. 3D culture models of Alzheimer's disease: a road map to a "cure-in-a-dish".Molecular Neurodegeneration. $2016 ; 75$.

16. De Strooper B. Lessons from a failed $\gamma$-secretase Alzheimer trial. Cell. 2014;159:721-6.

17. Mariani J, Coppola G, Zhang P, Abyzov A, Provini L, Tomasini $\mathrm{L}$ et al. FOXG1-dependent dysregulation of GABA/glutamate neuron differentiation in autism spectrum disorders. Cell .2015; 162: 375-90.

18. Jessica Hoarau-Véchot, Arash Rafii, Cyril Touboul, Jennifer Pasquier. Halfway between 2D and Animal Models: Are 3D Cultures the Ideal Tool to Study Cancer-Microenvironment Interactions. Int J Mol Sci. 2018;19:181.

19. Ruggeri BA, Camp F, Miknyoczki S. Animal models of disease: pre-clinical animal models of cancer and their applications and utility in drug discovery. Biochem Pharmacol. 2014; 87:150-61.

20. Clevers H. Modeling development and disease with organoids. Cell 2016; 165:1586-97.

21. Liu B, Li S, Hu J: Technological advances in high-throughput screening. Am J Pharmacogenomics 2004;4:263-76.

22. Booij, Tijmen H., et al. "3D Cell-Based Assays for Drug Screens: Challenges in Imaging, Image Analysis, and HighContent Analysis." SLAS DISCOVERY: Advancing Life Sciences R\&D; 2019.

23. Van den Brand D., Massuger L. F., Brock R., et al. Mimicking Tumors: Toward More Predictive in Vitro Models for Peptideand Protein-Conjugated Drugs. Bioconjug. Chem. 2017;846-56.

24. Fujii M, Shimokawa M, Date S, et al. A colorectal tumor organoid library demonstrates progressive loss of niche factor requirements during tumorigenesis. CellStem Cell 2016; 18: 827-38.

25. Lancaster M. A., Knoblich J. A. Organogenesis in a Dish: Modeling Development and Disease Using Organoid Technologies. Science. 2014;124-5.
26. Schwartz M. P, Hou Z, Propson N. E, et al. Human Pluripotent Stem Cell-Derived Neural Constructs for Predicting Neural Toxicity. Proc. Natl. Acad. Sci. U.S.A. 2015:12516-21.

27. Gunness P., Mueller D., Shevchenko V., et al. 3D Organotypic Cultures of Human HepaRG Cells: A Tool for In Vitro Toxicity Studies. Toxicol. Sci.2013:67-78.

28. Siramshetty VB, Nickel J, Omieczynski C, Gohlke BO, Drwal $\mathrm{MN}$, Preissner R.WITHDRAWN -a resource for withdrawn and discontinued drugs. Nucleic Acids Res 2016; 44(D1): D1080-6.

29. Takasato M, Er PX, Chiu HS, et al. Kidneyorganoids from human iPS cells contain multiple lineages and model human nephrogenesis. Nature 2015; 526: 564-8.

30. Li Z, Araoka T, Wu J, Liao HK2, Li M1, Lazo M, et al. 3D culture supports long-term expansion of mouse and human nephrogenic progenitors. Cell Stem Cell 2016; 19:516-29.

31. Esch E, Bahinski A., Huh D. Organs-on-Chips at the Frontiers of Drug Discovery. Nat. Rev. Drug Discov. 2015:14;248-60

32. Huh D, Leslie D.C., Matthews B. D, Fraser JP, Jurek S, Hamilton GA, et al. A Human Disease Model of Drug Toxicity-Induced Pulmonary Edema in a Lung-on-a-Chip Microdevice. Sci. Transl. Med. 2012; 4, 159 ra147.

33. Schwank G, Koo BK, Sasselli V, Dekkers J.F, Heo I, Demircan $\mathrm{T}$ et al. Functional repair of CFTR by CRISPR/Cas9 in intestinal stem cell organoids of cystic fibrosis patients. Cell Stem Cell. 2013;13:653-8.

34. Huch M., Knoblich J.A., Lutolf M.P., Martinez-Arias A. The hope and the hype of organoid research. Development. 2017;144:938-41.

35. Yin X., Mead B.E., Safaee H., Langer R., Karp J.M., Levy O. Engineering stem cell organoids. Cell Stem Cell. 2016;18:2538.

36. Ley K, Laudanna C, Cybulsky M.I, Nourshargh S. Getting to the site of inflammation: The leukocyte adhesion cascade updated. Nat. Rev. Immunol.2007;7:678-89.

Table 1: 3D cell culture organoids vs. $2 \mathrm{D}$ cell culture models vs. animal models

\begin{tabular}{llll} 
Functions & 3D Cell culture organoids & 2D cell cultures & Animal models \\
Physiologic enactment & Semi physiologic & Limited & Physiologic \\
Human development and disease modeling & Yes & Poor & Yes \\
High-throughput screening & Yes & Yes & No \\
Manageability & $\begin{array}{l}\text { Good, may have some experimental } \\
\text { variability }\end{array}$ & Excellent & Limited \\
Organogenesis modeling & $\begin{array}{l}\text { Suitable for the study of cell-cell com- } \\
\text { munication, morphogenesis; }\end{array}$ & poor & Yes \\
Vascularization and immune system & No & No & Yes \\
cost & Less & Less & High \\
\hline
\end{tabular}




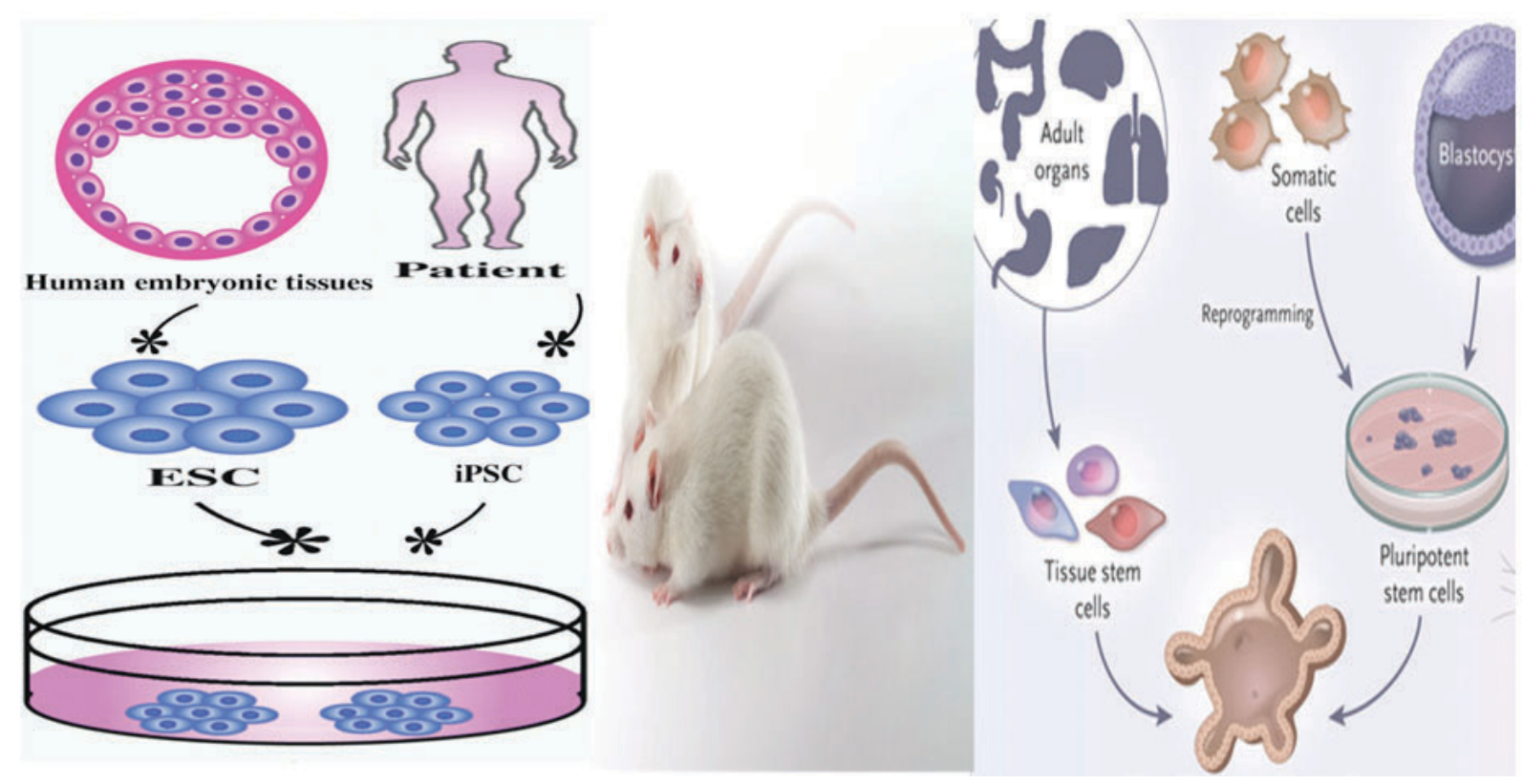

Figure 1: 3D cell culture organoids vs. 2D cell culture models vs. animal models. 